\title{
Percepção da funcionalidade nas fases leve e moderada da doença de Alzheimer: visão do paciente e seu cuidador
}

\author{
Perception of functionality in mild and moderate stages of Alzheimer's disease: vision of the \\ patient and their caregiver
}

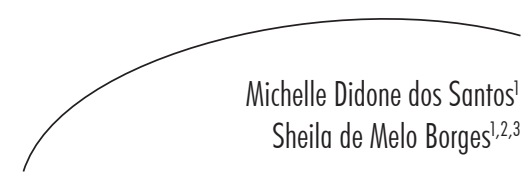

\section{Resumo}

Objetivos: Avaliar e comparar a percepção da funcionalidade de idosos com doença de Alzheimer (DA) em relação à percepção de seus cuidadores, bem como avaliar e comparar essa relação de acordo com o grau de comprometimento cognitivo desses idosos. Métodos: Foi realizado estudo transversal em 20 idosos com DA e seus cuidadores, sendo aplicada a Medida de Independência Funcional (MIF) para avaliar a percepção da funcionalidade de acordo com o relato do idoso com DA e seu cuidador; foi aplicada a escala de avaliação clínica da demência (CDR) para avaliar o estadiamento clínico da demência, sendo considerados idosos com DA leve (CDR 1) e DA moderada (CDR 2). Resultados: Comparada a percepção da funcionalidade da amostra total de idosos com DA e seus respectivos cuidadores, a média da MIF foi de 116,5 ( $\mathrm{dp}=9,8)$ pontos, segundo a visão dos idosos, e a média foi de $88,5(\mathrm{dp}=19,0)$ pontos nas tarefas avaliadas pela MIF, de acordo com seus cuidadores $(p<0,001)$. O mesmo padrão foi observado na comparação entre os idosos com DA leve e seus cuidadores, embora os itens "autocuidados" e "mobilidade da MIF" não tenham sido estatisticamente significativos. Já nos idosos com DA moderada, houve diferença significativa em todas as dimensões da MIF quando comparada a seus cuidadores. Conclusão: Idosos com DA subestimam suas dificuldades quando comparados com a percepção de seus cuidadores e, à medida que a gravidade da doença aumenta, há piora progressiva da percepção da sua funcionalidade.

\section{Abstract}

Objectives: Evaluate and compare of the perception of functionality in elderly with Alzheimer's disease (AD) with the functionality they present according with the perception of their caregivers, as well as evaluate this relationship according to the degree of cognitive problems in this population. Methods: Cross-sectional study in 20

\footnotetext{
Universidade Santa Cecília, Curso Fisioterapia. Santos, SP, Brasil.

2 Universidade Santa Cecília, Ciências Biológicas e Saúde, Programa de Pós-graduação em Gerontologia Integrativa. Santos, SP, Brasil.

3 Universidade Santa Cecília, Ciências Biológicas e Saúde, Programa de Pós-graduação em Fisioterapia Neurofuncional. Santos, SP, Brasil.
}

Palavras-chave: Doença de Alzheimer. Cuidadores. Percepção. Percepção da Funcionalidade. 
older people with AD and their caregivers, applying the Functional Independence Measures (FIM) to evaluate the perception of functionality according to the report of the elderly with AD and their caregiver; it was used the Clinical Dementia Rating Scale (CDR) to evaluate the clinical staging of the dementia, considering elderly with mild AD (CDR 1) and moderate AD (CDR 2). Results: When comparing the perception of the functionality of the total sample of older person with $\mathrm{AD}$ and their caregivers, the mean of FIM was 116.5 ( $\mathrm{sd}=9.8$ ) points, reported by patients with $\mathrm{AD}$, and mean of 88.5 ( $\mathrm{sd}=19.0)$ points according to their caregivers $(p<0.001)$. The same pattern was observed in the comparison between the elderly with mild AD and their caregivers, although self-care and mobility FIM's items have not been statistically significant. In the elderly with moderate $\mathrm{AD}$, there were significant differences in all dimensions of the FIM, when compared to their caregivers. Conclusion: Older patients with AD underestimate their difficulties as compared to the perception of their caregivers and this perception of functionality is worse according with progression of AD.
Key words: Alzheimer

Disease. Caregivers.

Functionality. Perception of

Functionality.

\section{INTRODUÇÃO}

O envelhecimento populacional vem crescendo em todo o mundo durante as últimas décadas. Esse aumento significativo de idosos traz como consequência um maior número de pessoas afetadas por doenças, dentre as quais se destaca a doença de Alzheimer (DA), ${ }^{1}$ um dos mais importantes problemas de saúde pública da atualidade. ${ }^{2,3}$

A DA está relacionada à perda cognitiva progressiva, que leva ao declínio funcional e perda gradual de autonomia, que, em consequência, ocasionam dependência total de outras pessoas. Também se destaca por representar de 50 a $60 \%$ do número total de casos de demência, acometendo aproximadamente 10 a $20 \%$ dos indivíduos com mais de 65 anos de idade. ${ }^{4,5}$ Os mecanismos patológicos responsáveis por essa doença ainda permanecem em grande parte desconhecidos. Os principais achados são perda neuronal, degeneração sináptica intensa e aumento significativo da deposição de placas senis e emaranhado neurofibrilares no córtex cerebral. ${ }^{6}$ A perda da memória é, em geral, o sintoma mais proeminente e precoce, podendo causar grande impacto nas atividades de vida diária (AVDs), sendo a capacidade funcional considerada um novo paradigma de saúde para o idoso., ${ }^{7,8}$

Conforme o avançar do comprometimento cognitivo na DA, esses idosos necessitam gradativamente de ajuda para realizar suas AVDs, ou seja, precisam de uma pessoa que cuide deles e execute as tarefas do cotidiano que o idoso não consegue mais realizar. ${ }^{9,10} \mathrm{Na}$ fase leve, esses idosos podem ter perda da memória recente, desorientação temporal e espacial e apresentar perda do interesse por hobbies e outras atividades. Já na fase moderada, as dificuldades ficam mais evidentes e severas; há o esquecimento de nomes de pessoas, dificuldade em viver sozinho devido à incapacidade de realizar atividades mais complexas, como fazer compras, cozinhar e cuidar da casa, sinais de irritabilidade e alucinações, além de dificuldade na fala. $\mathrm{Na}$ fase grave, o prejuízo da memória encontra-se bastante comprometido, há dificuldade na deglutição e de orientar-se dentro de sua própria casa, incontinência urinária/fecal e prejuízo na marcha, podendo posteriormente necessitar de cadeira de rodas ou ficar acamado. ${ }^{11}$

Além da perda de independência e de autonomia, e da necessidade maior de cuidados associados ao avanço da DA, esses idosos podem apresentar comprometimento da consciência, esta definida como a capacidade de perceber em si e/ ou nas AVDs alterações ocasionadas por déficits associados ao processo de adoecimento. ${ }^{12,13}$ Esses distúrbios na percepção são chamados de "anosognosia" e podem afetar diretamente o desempenho das funções cotidianas. Além disso, é sabido que a percepção, bem como reconhecimento de uma informação do ambiente e/ou do meio interno, é a base da cognição. ${ }^{14,15}$ 
Com base em revisão de literatura sobre a temática, constatou-se que há escassez de trabalhos científicos que tratam especificadamente da percepção do paciente em relação a seus déficits, sendo importante a realização de pesquisas que acrescentem informações e conhecimento sobre essa relação. Nesse sentido, destacam-se os estudos de Almeida \& Crocco, ${ }^{2}$ Sousa et al. ${ }^{13}$ e Dourado et al., ${ }^{16}$ que observaram que os idosos subestimam suas dificuldades e que uma entrevista com o cuidador pode fornecer informações mais precisas quanto a suas dificuldades.

Dessa maneira, a presente pesquisa pode contribuirparaavaliarograudecomprometimento cognitivo e sua relação com a percepção funcional dos idosos com diagnóstico de DA, bem como analisar essa relação segundo a percepção de seus cuidadores (que os acompanham e identificam suas necessidades diárias), facilitando assim as orientações de cuidado, respeitando a autonomia desses idosos, contribuindo para a manutenção de sua independência funcional por meio de intervenção específica (avaliação e tratamento adequados) multi/interdisciplinar, visando ao atendimento das reais dificuldades e limitações dos idosos com DA. Além disso, pretendese contribuir cientificamente para um melhor entendimento sobre essa doença, por se tratar de uma relação ainda pouco explorada na literatura científica nacional ${ }^{12,13,16} \mathrm{e}$ internacional..$^{14,17-19}$

O presente estudo teve por objetivo avaliar e comparar a percepção da funcionalidade de idosos com doença de Alzheimer (DA) em relação à percepção de seus cuidadores, bem como avaliar e comparar essa relação de acordo com o grau de comprometimento cognitivo (leve e moderado) desses idosos.

\section{MÉTODOS}

Trata-se de estudo analítico, observacional, do tipo corte transversal, que avaliou 20 idosos com diagnóstico de DA e seus cuidadores $(n=20)$, que frequentavam o Centro de Referência do Idoso do Estado de São Paulo - Instituto Paulista de Geriatria e Gerontologia - José Ermírio de Moraes (IPGG), no período de janeiro e fevereiro de 2014.
Os critérios de inclusão dos participantes no estudo foram: para os idosos: a) idade igual ou superior a 60 anos; b) ambos os gêneros; c) ter diagnóstico de DA; d) ter necessidade de um cuidador responsável; e) ter escores sugestivos de déficit cognitivo de acordo com o teste de rastreio do Miniexame do Estado Mental (MEEM) segundo nota de corte por escolaridade (20 para analfabetos; 1-4 anos de escolaridade: $25 ; 5-8$ anos: 26; 9-11 anos: 28; >11 anos: 29 pontos); ${ }^{20} \mathrm{f}$ ) demência leve (CDR 1) ou demência moderada (CDR 2) considerada pelo Estadiamento Clínico das demências (CDR). ${ }^{21}$ Para os cuidadores: a) idade igual ou superior a 18 anos; b) ser o acompanhante do idoso na consulta/tratamento no IPGG; c) ser o responsável pelo cuidado do idoso em suas AVDs; d) ter contato com o idoso pelo menos quatro dias por semana.

Após consulta de rotina com o médico geriatra, os idosos e seus cuidadores foram convidados a participar da pesquisa no próprio IPGG. Aqueles que concordaram em participar foram entrevistados individualmente pela autora principal do estudo, que foi previamente treinada para aplicar o protocolo de pesquisa (detalhado a seguir), sendo as informações transcritas, não havendo necessidade de gravações.

Para caracterização da amostra, foi aplicado um questionário contendo informações pessoais e dados sociodemográficos, tais como: nome, data de nascimento, idade, gênero, escolaridade, e grau de parentesco (sendo essa última pergunta aplicada e direcionada apenas aos cuidadores). Posteriormente, foi aplicada a Medida de Independência Funcional (MIF) em relação à ajuda fornecida pelos cuidadores para a realização das atividades funcionais dos idosos avaliados, esta considerada como a percepção do cuidador.

A avaliação dos idosos deu-se da seguinte maneira: preenchimento de uma ficha de caracterização sociodemográfica com confirmação dos dados pelo cuidador (uma vez que algumas informações podem não corresponder à realidade, em decorrência do comprometimento cognitivo - por exemplo, idade/data de nascimento); rastreamento cognitivo por meio do MEEM e estadiamento 
da doença por meio do CDR; e por fim, foi avaliada a percepção da sua capacidade funcional por meio da MIF.

O MEEM é um instrumento de rastreamento cognitivo que avalia orientação temporal, orientação espacial, registro de três palavras, atenção e cálculo, recordação das três palavras, linguagem e capacidade construtiva visual. O escore pode variar de um mínimo de 0 ponto, que indica o maior grau de comprometimento cognitivo dos indivíduos, até um total máximo de 30 pontos, o qual, por sua vez, corresponde à melhor capacidade cognitiva, sendo utilizadas as notas de corte propostas no estudo de Brucki et al. ${ }^{20}$

A CDR é uma escala de avaliação do estadiamento da demência que varia de 0 (CDR 0 - sem demência) a 3 (CDR 3 - demência grave), de acordo com memória, orientação, julgamento e solução de problemas, assuntos na comunidade, lar e passatempos e cuidados pessoais. ${ }^{21}$ No estudo, foram aceitos apenas idosos com demência leve (CDR 1) e demência moderada (CDR 2), a fim de evitar possíveis vieses, uma vez que idosos com demência grave (CDR 3) poderiam não compreender o que estava sendo perguntado, além de terem dificuldade em responder, devido ao avanço da doença.

A MIF é constituída por 18 itens, cada qual tendo a pontuação máxima de 7 (independência completa), e cotação mínima de 1 (dependência total), onde se deve responder a questões relacionadas às atividades de vida diária. Os aspectos verificados na avaliação são: autocuidados, controle de esfíncteres, transferências, locomoção, comunicação e cognição social. ${ }^{22}$ Somando-se todos os escores, obtêm-se escore total mínimo de 18 e o máximo de 126 pontos, que caracterizam os níveis de dependência entre todos os itens. Sendo eles: 18 pontos - dependência completa; 19 a 60 pontos - dependência modificada (assistência de até $50 \%$ da tarefa); 61 a 103 pontos - dependência modificada (assistência de até $25 \%$ da tarefa); e 104 a 126 pontos - independência completa/ modificada. $^{23}$
Os dados coletados foram analisados por meio do programa SPSS 18.0, sendo as variáveis numéricas expressas em média e desvio-padrão (dp) e as variáveis categóricas expressas com frequência absoluta e relativa.

Para análise comparativa entre os idosos (tanto amostra total, como de acordo com a gravidade da DA - CDR 1 e CDR 2) e entre seus cuidadores, foram utilizados os testes Exato de Fisher para dados categóricos, e Mann-Whitney e $t$ Student para amostras independentes para os dados numéricos, conforme resultado do teste de normalidade. O nível de $5 \%(p<0,05)$ foi considerado para obtenção de significância estatística.

O estudo foi conduzido de acordo com as diretrizes e normas regulamentadoras de pesquisas envolvendo seres humanos (Resolução $n^{\circ}$ 466/12, do Conselho Nacional de Saúde), tendo sido obtida aprovação do IPGG e do Comitê de Ética em Pesquisa da Universidade Santa Cecília, deste último em 19/11/2013 com o CAAE no 23683413.0.0000.5513. Por tratarse de idosos com diagnóstico de DA e avaliação de seus cuidadores, ambos foram convidados a participar da pesquisa e assinar o Termo de Consentimento Livre e Esclarecido (TCLE), mas no caso de idosos e/ou cuidadores sem escolaridade (analfabetos), foram solicitadas suas assinaturas dactiloscópicas.

\section{RESULTADOS}

Os idosos com DA avaliados neste estudo apresentaram média de 81,2 anos ( $\mathrm{dp}=7,4)$; predominância do sexo feminino $(\mathrm{n}=17 ; 85 \%)$; média de 4,7 anos ( $\mathrm{dp}=2,6)$ de escolaridade; 12,6 pontos $(\mathrm{dp}=5,3)$ no MEEM, sendo sete idosos $(35 \%)$ em fase leve (CDR 1) e 13 idosos (65\%) em fase moderada da DA (CDR 2) (tabela 1). $\mathrm{Na}$ tabela 1, é possível observar que houve diferença significativa em relação à idade $(p=0,01)$, não sendo observada diferença nas demais variáveis (gênero, escolaridade e MEEM) entre os idosos com CDR 1 e CDR 2. 
Tabela 1. Caracterização da amostra total de idosos com doença de Alzheimer (DA) e comparação segundo classificação de DA leve (CDR 1) e moderada (CDR 2). São Paulo-SP, 2014.

\begin{tabular}{|c|c|c|c|c|c|}
\hline & & $\begin{array}{c}\text { DA total* } \\
\mathrm{n}=20\end{array}$ & $\begin{array}{c}\text { CDR } 1 \\
n=7\end{array}$ & $\begin{array}{c}\text { CDR } 2 \\
\mathrm{n}=13\end{array}$ & $p$-valor \\
\hline Idade & & $81,2 \pm 7,4$ & $75,7 \pm 5,2$ & $84,1 \pm 6,8$ & $0,01^{\mathrm{a}}$ \\
\hline Gênero & $\begin{array}{l}\text { Feminino } \\
\text { Masculino }\end{array}$ & $\begin{array}{c}17(85 \%) \\
3(15 \%)\end{array}$ & $\begin{array}{l}6(86 \%) \\
1(14 \%)\end{array}$ & $\begin{array}{c}11(85 \%) \\
2(15 \%)\end{array}$ & $1,00^{\mathrm{b}}$ \\
\hline Escolaridade & & $4,7 \pm 2,6$ & $6,0 \pm 2,6$ & $4,0 \pm 2,4$ & $0,10^{a}$ \\
\hline \multirow[t]{2}{*}{ CDR } & CDR 1 & $7(35 \%)$ & - & - & - \\
\hline & CDR 2 & $13(65 \%)$ & - & - & \\
\hline MEEM & & $12,6 \pm 5,3$ & $15,4 \pm 4,8$ & $11,1 \pm 5,1$ & $0,08^{\mathrm{a}}$ \\
\hline
\end{tabular}

Valores numéricos representados por média $\_$desvio-padrão e número absoluto ( $\left.\%\right)$; $a=p$-valor referente ao teste $t$ Student para amostras independentes na comparação entre os idosos CDR 1 e CDR 2; b= $p$-valor referente ao teste de Fisher na comparação entre os idosos CDR 1 e CDR 2; *A amostra de DA total é composta tanto por idosos com CDR 1 como CDR 2; CDR= escala de avaliação clínica da demência; CDR $1=$ demência leve; CDR 2= demência moderada; MEEM= Miniexame do estado mental.

Com relação à caracterização dos cuidadores dos idosos com DA, observa-se na tabela 2 predominância de mulheres ( $\mathrm{n}=17 ; 85 \%$ ), com idade média de 56,8 ( $\mathrm{dp}=11,9$ anos), sendo mais da metade ( $\mathrm{n}=12 ; 60 \%$ ) da amostra composta por filhas, seguidas de cônjuges ( $\mathrm{n}=4 ; 20 \%)$, nora $(\mathrm{n}=1 ; 5 \%)$ e irmã $(\mathrm{n}=1 ; 5 \%)$. É possível observar que dois cuidadores $(10 \%)$ não tinham nenhum grau de parentesco com o idoso, sendo um amigo da família e outro um cuidador profissional. Além disso, não houve diferença significativa em relação às características dos cuidadores de idosos com DA leve (CDR 1), em relação aos cuidadores com DA moderada (CDR 2) (tabela 2).

Tabela 2. Caracterização de cuidadores dos idosos com DA e comparação segundo classificação de DA leve (CDR 1) e moderada (CDR 2). São Paulo-SP, 2014.

\begin{tabular}{|c|c|c|c|c|c|}
\hline & & $\begin{array}{l}\text { Cuidadores } \\
\text { DA total* } \\
n=20\end{array}$ & $\begin{array}{c}\text { Cuidadores } \\
\text { CDR } 1 \\
n=7\end{array}$ & $\begin{array}{c}\text { Cuidadores } \\
\text { CDR } 2 \\
n=13\end{array}$ & $p$-valor \\
\hline Idade & & $56,8 \pm 11,9$ & $57,7 \pm 13,2$ & $56,2 \pm 11,7$ & $0,79^{\mathrm{a}}$ \\
\hline Gênero & $\begin{array}{l}\text { Feminino } \\
\text { Masculino }\end{array}$ & $\begin{array}{c}17(85 \%) \\
3(15 \%)\end{array}$ & $\begin{array}{l}6(86 \%) \\
1(14 \%)\end{array}$ & $\begin{array}{c}11(85 \%) \\
2(15 \%)\end{array}$ & $1,00^{\mathrm{b}}$ \\
\hline Parentesco & $\begin{array}{l}\text { Filha } \\
\text { Cônjuge } \\
\text { Nora } \\
\text { Irmã } \\
\text { Nenhum }\end{array}$ & $\begin{array}{c}12(60 \%) \\
4(20 \%) \\
1(5 \%) \\
1(5 \%) \\
2(10 \%)\end{array}$ & $\begin{array}{c}3(43 \%) \\
2(29 \%) \\
0(0 \%) \\
1(14 \%) \\
1(14 \%)\end{array}$ & $\begin{array}{l}9(69 \%) \\
2(15 \%) \\
1(8 \%) \\
0(0 \%) \\
1(8 \%)\end{array}$ & $0,47^{\mathrm{b}}$ \\
\hline
\end{tabular}

Valores numéricos representados por média \pm desvio-padrão e número absoluto (\%); $\mathrm{a}=p$-valor referente ao teste $t$ Student para amostras independentes na comparação entre os idosos CDR 1 e CDR 2; b= $p$-valor referente ao teste de Fisher na comparação entre os idosos CDR 1 e CDR 2; *A amostra de DA total é composta tanto por cuidadores de idosos com CDR 1 como cuidadores de idosos com CDR 2; CDR= escala de avaliação clínica da demência. 
Combase nos resultados encontrados nas cinco dimensões que compõem a MIF e sua pontuação total, observa-se na tabela 3 uma diferença significativa entre a percepção dos idosos com DA em relação à percepção dos seus cuidadores. A média do escore total da MIF segundo a percepção do idoso foi de 116,5 (dp=9,8) pontos, indicando independência completa/modificada, já a percepção dos cuidadores em relação aos idosos foi de 88,5 ( $\mathrm{dp}=19,0)$ pontos, indicando dependência modificada, com assistência de até $25 \%$ da tarefa $(p<0,001)$.

Tabela 3. Comparação entre a percepção da funcionalidade dos idosos com DA e seus cuidadores. São Paulo-SP, 2014.

\begin{tabular}{lccc}
\hline $\begin{array}{c}\text { Dimensão da MIF } \\
\text { [pontuação esperada] }\end{array}$ & $\begin{array}{c}\text { DA total* } \\
\mathrm{n}=20\end{array}$ & $\begin{array}{c}\text { Cuidador total } \\
\mathrm{n}=20\end{array}$ & $p$-valor \\
\hline Autocuidados [6-42] & $38,1 \pm 5,5$ & $30,6 \pm 9,1$ & $\mathbf{0 , 0 0 1}$ \\
Controle de esfíncteres [2-14] & $13,2 \pm 1,2$ & $10,5 \pm 2,5$ & $<\mathbf{0 , 0 0 1}$ \\
Mobilidade [5-35] & $30,6 \pm 5,0$ & $24,6 \pm 5,4$ & $\mathbf{0 , 0 0 1}$ \\
Comunicação [2-14] & $13,8 \pm 0,7$ & $11,2 \pm 1,9$ & $<\mathbf{0 , 0 0 1}$ \\
Cognição social [3-21] & $19,2 \pm 1,5$ & $12,0 \pm 3,6$ & $<\mathbf{0 , 0 0 1}$ \\
MIF total [18-126] & $116,5 \pm 9,8$ & $88,5 \pm 19,0$ & $<\mathbf{0 , 0 0 1}$ \\
\hline
\end{tabular}

Média \pm desvio-padrão com $p$-valor referente ao teste Mann-Whitney; *A amostra de DA total é composta tanto por idosos com CDR 1 como CDR 2; MIF= medida de independência funcional.

De acordo com o grau de comprometimento cognitivo, os idosos CDR 1 (DA leve) referiram melhor capacidade funcional quando comparados à percepção de seus cuidadores na pontuação total da MIF $(p=0,025)$ e nas dimensões da MIF: controle de esfincteres $(p=0,010)$, comunicação $(p=0,025)$ e cognição social $(p=0,005)$; não sendo observada diferença significativa nas dimensões autocuidados e mobilidade (tabela 4).

Idosos com DA moderada (CDR 2) relataram melhor desempenho na MIF total e em todas as dimensões da MIF, em comparação à percepção de seus cuidadores, sendo essas diferenças estatisticamente significativas, conforme a tabela 5 .

Tabela 4. Comparação entre a percepção da funcionalidade dos idosos com DA leve (CDR 1) e seus cuidadores. São Paulo-SP, 2014.

\begin{tabular}{lccc}
\hline \multicolumn{1}{c}{$\begin{array}{c}\text { Dimensão da MIF } \\
\text { [pontuação esperada] }\end{array}$} & $\begin{array}{c}\text { Idoso } \\
\mathrm{n}=7\end{array}$ & $\begin{array}{c}\text { Cuidador } \\
\mathrm{n}=7\end{array}$ & $p$-valor \\
\hline Autocuidados [6-42] & $38,6 \pm 4,0$ & $35,4 \pm 5,7$ & $0,261^{\mathbf{a}}$ \\
Controle de esfíncteres [2-14] & $13,6 \pm 0,8$ & $11,6 \pm 1,7$ & $\mathbf{0 , 0 1 0 ^ { \mathbf { b } }}$ \\
Mobilidade [5-35] & $29,9 \pm 5,0$ & $26,9 \pm 4,6$ & $0,261^{\mathbf{a}}$ \\
Comunicação [2-14] & $14,0 \pm 0,0$ & $12,7 \pm 1,4$ & $\mathbf{0 , 0 2 5 ^ { \mathbf { b } }}$ \\
Cognição social [3-21] & $18,7 \pm 1,1$ & $14,1 \pm 3,7$ & $\mathbf{0 , 0 0 5 ^ { \mathbf { b } }}$ \\
& $116,4 \pm 8,4$ & $99,9 \pm 15,0$ & $\mathbf{0 , 0 2 5 ^ { \mathbf { a } }}$ \\
\hline
\end{tabular}

Média \pm desvio-padrão, sendo: $\mathrm{a}=p$-valor referente ao teste $t$ Student para amostras independentes; $\mathrm{b}=p$-valor referente ao teste Mann-Whitney; $\mathrm{MIF}=$ medida de independência funcional. 
Tabela 5. Comparação entre a percepção da funcionalidade dos idosos com DA moderada (CDR 2) e seus cuidadores. São Paulo-SP, 2014.

\begin{tabular}{lccc}
\hline \multicolumn{1}{c}{$\begin{array}{c}\text { Dimensão da MIF } \\
\text { [pontuação esperada] }\end{array}$} & $\begin{array}{c}\text { Idoso } \\
\mathrm{n}=13\end{array}$ & $\begin{array}{c}\text { Cuidador } \\
\mathrm{n}=13\end{array}$ & $p$-valor \\
\hline Autocuidados [6-42] & $37,9 \pm 6,3$ & $28,0 \pm 9,8$ & $<\mathbf{0 , 0 0 1}$ \\
Controle de esfíncteres [2-14] & $12,9 \pm 1,4$ & $9,9 \pm 2,8$ & $\mathbf{0 , 0 0 2}$ \\
Mobilidade [5-35] & $31,0 \pm 5,1$ & $23,4 \pm 5,7$ & $\mathbf{0 , 0 0 3}$ \\
Comunicação [2-14] & $13,7 \pm 0,9$ & $10,3 \pm 1,6$ & $<\mathbf{0 , 0 0 1}$ \\
Cognição social [3-21] & $19,5 \pm 1,7$ & $10,8 \pm 3,1$ & $<\mathbf{0 , 0 0 1}$ \\
MIF total [18-126] & $116,5 \pm 10,8$ & $82,3 \pm 18,7$ & $<\mathbf{0 , 0 0 1}$ \\
\hline
\end{tabular}

Média \pm desvio-padrão com $p$-valor referente ao teste Mann-Whitney; MIF= medida de independência funcional.

\section{DISCUSSÃO}

No presente estudo, os idosos com DA apresentaram, em média, idade acima dos 80 anos, com predominância do sexo feminino, o que está de acordo com a literatura. ${ }^{8,24,25}$ Esse dado reforça a característica de uma doença idade dependente ${ }^{1}$ e pode sofrer influência da feminilização da velhice, uma vez que as mulheres podem ser mais afetadas pela doença devido ao fato de viverem mais que os homens. ${ }^{26}$ Outro importante fator de risco para DA é a baixa escolaridade, ${ }^{20,25}$ sendo esse dado observado neste estudo com uma média de 4,7 anos de escolaridade na população estudada.

Ao analisar os idosos CDR 1 e CDR 2, observa-se que a única diferença em relação à caracterização da amostra diz respeito à idade, com média de 84,1 anos nos idosos com CDR 2 em relação aos idosos com CDR 1, com média de 75,7 anos, corroborando o estudo de Dourado et al., ${ }^{16}$ que também observou que idosos com CDR 2 eram estatisticamente mais velhos em comparação aos idosos com CDR 1. Esse dado pode demonstrar em parte que, além de ser uma doença associada ao envelhecimento, é uma doença muito mais comum em idosos considerados velhos e muito velhos. ${ }^{27}$ Além disso, com o avanço da DA, os idosos passam a ficar cada vez mais dependente dos familiares, necessitando de um cuidador que fique responsável por auxiliá-los em suas AVDs. ${ }^{10}$

Em países como o Brasil, 90\% dos cuidadores são membros da família devido à dificuldade financeira em manter um apoio formal e quem desempenha essa tarefa geralmente é a mulher de meia idade, por caber aos filhos e cônjuges cuidar desses idosos. ${ }^{9,10} \mathrm{O}$ perfil dos cuidadores avaliados no estudo está de acordo com essas pesquisas e com outras encontradas na literatura, ${ }^{28,29}$ onde foi possível observar que a maioria dos cuidadores era do sexo feminino, com maior frequência de filhas e cônjuges. Esse fato pode estar associado a um padrão cultural no qual cabe à mulher a organização da vida familiar, sendo visto o cuidado como uma função feminina. No início da doença, esses cuidadores se responsabilizam por atividades instrumentais de vida diária, como cuidar das finanças desses idosos e à medida que a doença progride, o cuidado passa a ser mais intenso, tendo que auxiliar em cuidados mais básicos, como por exemplo, higiene pessoal, banho e alimentação. ${ }^{28}$

Sabe-se que, independentemente das condições de risco para o desenvolvimento da DA, o tempo de avanço da demência varia de pessoa para pessoa, mas que além do comprometimento cognitivo, a capacidade funcional é um dos marcadores da evolução da doença. ${ }^{11}$ Os idosos 
com DA avaliados nesta pesquisa relataram melhor desempenho nas dimensões avaliadas na MIF em relação a seus cuidadores, sendo essa relação ainda mais evidenciada quando analisada segundo o avanço do comprometimento cognitivo (idosos com CDR2).

Foi possível observar, na presente pesquisa, que quando comparadas as respostas dos idosos com DA total (tanto CDR 1 quanto CDR 2) e seus cuidadores, a média foi significativamente maior em todas as dimensões da MIF dos idosos, demonstrando que os idosos em questão subestimam suas dificuldades. Isso evidencia que uma entrevista com seu cuidador pode fornecer informações mais reais a respeito da gravidade da doença, o que corrobora o estudo de Almeida \& Crocco. ${ }^{2}$ Os autores avaliaram a concordância de idosos e cuidadores por meio da aplicação do questionário de demência-ampliada, e ao final concluiu-se que os idosos subestimaram seus déficits na maioria das questões propostas.

Quando comparadas as dimensões da MIF apenas dos idosos CDR 1, apesar de apresentarem médias significativamente maiores na maioria das dimensões avaliadas em comparação à percepção de seus cuidadores, as dimensões "autocuidados" e "mobilidade" não foram estatisticamente significativas. Esse fato pode estar associado a uma possível preservação tanto da sua independência nessas AVDs, como em relação a sua percepção de necessidade de cuidados nessas atividades. É esperado que, na fase inicial da $\mathrm{DA}$, os idosos ainda não sejam completamente dependentes para realizar suas atividades funcionais. ${ }^{10}$

$\mathrm{Na}$ DA, segundo a teoria da retrogênese ("involução" humana), a funcionalidade seguiria o inverso da sua aquisição do desenvolvimento psicomotor conquistado durante a ontogênese (evolução humana). Dessa maneira, da mesma forma que a funcionalidade é adquirida conforme ocorre o desenvolvimento cognitivo na ontogênese, na DA observa-se uma perda da independência funcional diretamente relacionada com a progressão do prejuízo cognitivo. Dentre as atividades funcionais prejudicadas na fase inicial da DA, destaca-se a realização para atividades laborais, incontinência esfincteriana, comunicação, dentre outras. ${ }^{1}$

Cabe ressaltar que, no presente estudo, o item "controle de esfíncteres" mostrou-se significativo, e isso pode ocorrer por alterações do trato urinário relacionadas ao envelhecimento mesmo na ausência de demências, além disso, é frequente que os idosos subestimem as alterações urinárias por considerarem erroneamente essas alterações normais do processo de envelhecimento. ${ }^{30}$ Com relação à diferença entre a percepção da "comunicação" e "cognição social", por serem atividades que estão diretamente ligadas aos aspectos cognitivos, sendo esses intimamente relacionados à percepção das alterações cognitivas e da DA em si, essas dimensões corroboram a hipótese de anosognosia em estágios iniciais da DA. ${ }^{14,17,31}$

A hipótese de anosognosia relacionada ao avanço da DA é observada pela diferença significativa em todas as dimensões da MIF em idosos com DA moderada (CDR 2), em comparação a seus cuidadores, o que sugere que a percepção dos próprios déficits fica cada vez mais distante da realidade com a evolução da doença, sendo essa relação apontada em diversos estudos. ${ }^{13,14,18,19,31}$ Com isso, fazem-se necessárias ações educativas direcionadas aos cuidadores sobre o manejo adequado da doença e dos cuidados com sua própria saúde, tendo em vista que nem todas as famílias possuem membros preparados para assumir a responsabilidade de ser um cuidador, o que pode levar a uma sobrecarga física e emocional. ${ }^{28,29}$

Sousa et al. ${ }^{13}$ realizaram estudo longitudinal sobre a consciência da doença na DA, na qual, em um primeiro momento, alguns idosos CDR 1 apresentaram consciência da doença preservada ou parcial; em um segundo momento todos esses idosos haviam progredido para consciência da doença parcial ou ausente, e muitos deles evoluído para CDR 2. Os autores concluíram que há comprometimento significativo ao longo do percurso da doença. Kashiwa et al. ${ }^{18}$ observaram que o grau de anosognosia aumenta à medida que o idoso com DA se torna mais velho e as dificuldades em realizar AVDs aumentam, 
demonstrando que a anosognosia está fortemente associada ao progresso da doença.

Recente publicação ${ }^{32}$ mostrou que idosos com DA com CDR 1 apresentavam menor medo de cair e maior número de quedas em comparação aos idosos com comprometimento cognitivo leve (CDR 0,5) e idosos saudáveis cognitivamente. De acordo com essa pesquisa, o menor medo de cair associado ao maior número de quedas pode ocorrer devido a uma menor autocrítica e consciência de seus déficits em pacientes com DA. ${ }^{32}$ Dessa maneira, pode-se observar que são múltiplas as implicações da consciência da doença em idosos com DA, não apenas em relação à funcionalidade, que é o foco deste estudo, mas em relação ao medo de cair, por exemplo.

Frente aos resultados observados nesta pesquisa, pela relevância clínica e científica do assunto, espera-se que o estudo possa auxiliar a comunicação dos cuidadores com os idosos com DA. Por desconhecimento da doença, muitos cuidadores podem não entender as dificuldades desses idosos, o que pode dificultar a preservação da independência e autonomia deles, causar conflitos e gerar ansiedade e estresses de ambas as partes. ${ }^{33}$ Além disso, demonstra a importância de uma avaliação direcionada ao idoso, mas com a devida confirmação das informações em particular/reservadamente ou de forma discreta e respeitosa com seus cuidadores, que são pessoas responsáveis diariamente por esses idosos, uma vez que há evidências ${ }^{23}$ de que o relato de funcionalidade dos cuidadores está de acordo com a avaliação direta do profissional da área da saúde.

O presente estudo apresenta algumas limitações, tais como: pequeno tamanho amostral em ambos os grupos, especialmente no grupo com DA leve (CDR 1); e avaliação da percepção, por meio de relato (avaliação subjetiva) das atividades funcionais tanto pelos idosos quanto pelos cuidadores - ou seja, a funcionalidade não foi avaliada de forma ecológica (por observação direta da sua realização). Além disso, não foram avaliados outros fatores relacionados a aspectos neuropsicológicos dos idosos, tais como capacidade de julgamento, dificuldade de expressão e outros, que podem interferir no comprometimento da consciência da doença (anosognosia).

Vale ressaltar que ainda há dificuldade considerável no que diz respeito ao diagnóstico precoce da DA, ${ }^{11}$ e apesar da concordância descrita na literatura dos resultados da MIF por meio da observação das atividades realizadas pelo paciente e o relato do seu cuidador, ${ }^{23}$ o presente estudo sugere ampliação da pesquisa por meio de um maior número de idosos avaliados; estudos que avaliem diferentes condições patológicas (como por exemplo, comprometimento cognitivo leve, doença de Parkinson, pacientes com sequela de acidente vascular encefálico, etc.); e estudos comparativos entre avaliação ecológica da funcionalidade, bem como o relato do idoso e seu cuidador sobre essa temática e associados a testes neuropsicológicos mais específicos.

\section{CONCLUSÃO}

Com os resultados deste estudo, foi possível observar que os idosos com doença de Alzheimer apresentam pontuações maiores na Medida de Independência Funcional, quando comparados com a percepção de seus cuidadores a respeito de sua funcionalidade. $\mathrm{O}$ fato sugere que esses idosos subestimam suas dificuldades desde a fase leve da doença e, conforme aumenta o comprometimento cognitivo, há piora progressiva da consciência da percepção da sua funcionalidade, atingindo assim os objetivos do estudo.

Portanto, estudos como este levam a um melhor entendimento sobre a funcionalidade, percepção da doença de Alzheimer e a evolução desse tipo de demência, podendo facilitar o planejamento terapêutico dos profissionais de saúde. Informações mais precisas sobre as reais dificuldades desses idosos devem ser colhidas com seus respectivos cuidadores, de modo a facilitar uma intervenção correta, mantendo a autonomia e independência funcional o quanto for possível e proporcionando melhor qualidade de vida dessa população, apesar da evolução e progressão da doença. 


\section{REFERÊNCIAS}

1. Borges SM, Aprahamian I, Radanovic M, Forlenza O. Psicomotricidade e retrogênese: considerações sobre o envelhecimento e a doença de Alzheimer. Rev Psiquiatr Clín 2010;37(3):131-7.

2. Almeida OP, Crocco EI. Percepção dos déficits cognitivos e alterações do comportamento em pacientes com doença de Alzheimer. Arq Neuropsquiatr 2000;58(2A):292-9.

3. Intituto Brasileiro de Geografia e Estatística [Internet]. Rio de Janeiro: IBGE, [1995- ]. População brasileira envelhece em ritmo acelerado. Comunicação social; 2008 [acesso em 20 jul 2013]. Disponível em: http://www.ibge.gov.br/home/presidencia/noticias/ noticia_visualiza.php?id_noticia $=1272$.

4. Camarano AA. Envelhecimento da população brasileira: uma contribuição demográfica. In: Freitas EV, Py L, Neri AL, Cançado FAX, Gorzoni ML, Rocha SM, editores. Tratado de geriatria e gerontologia. Rio de Janeiro: Guanabara Koogan; 2002. p. 58.

5. Inouye K, Pedrazzani ES, Pavarini SCI, Toyoda CY. Percepção de qualidade de vida do idoso com demência e seu cuidador familiar: avaliação e correlação. Rev Latinoam Enferm 2009;17(2):187-93.

6. Machado JCB. Doença de Alzheimer. In: Freitas EV, Py L, Neri AL, Cançado FAX, Gorzoni ML, Rocha SM. Tratado de geriatria e gerontologia. Rio de Janeiro: Guanabara Koogan; 2002. p. 134-7.

7. Pereira LSM, Dias RC, Dias JMD, Gomes GC, Sitta MI. Fisioterapia. In: Freitas EV, Py L, Neri AL, Cançado FAX, Gorzoni ML, Rocha SM. Tratado de geriatria e gerontologia. Rio de Janeiro: Guanabara Koogan; 2002. p. 854.

8. Inouye K, Pedrazzani ES, Pavarini SCI. Influência da doença de Alzheimer na percepção de qualidade de vida do idoso. Rev Esc Enferm USP 2010;44(4):1093-99.

9. Lemos ND, Gazzola JM, Ramos LR. Cuidando do paciente com Alzheimer: o impacto da doença no cuidador. Saúde Soc 2006;15(3):170-9.

10. Falcão DVS, Maluschke JSNFB. Filhas que cuidam de pais/mães com provável/possível Doença de Alzheimer. Estud Psicol 2008;13(3):245-56.

11. Oliveira MF, Ribeiro M, Borges R, Luginger S. Doença de Alzheimer: perfil neuropsicológico e tratamento [Trabalho de Licenciatura]. Portugal: Universidade Lusíada do Porto; 2005.
12. Dourado M, Laks J, Leibing A, Engelhardt E. Consciência da doença na demência. Rev Psiquiatr Clín 2006;33(6):313-21.

13. Sousa MFB, Santos RL, Arcoverde C, Dourado M, Laks J. Consciência da doença na doença de Alzheimer: resultados preliminares de um estudo longitudinal. Rev Psiquiatr Clín 2011;38(2):57-60.

14. Starkstein SE, Jorge R, Mizhahi R, Robinson RG . A diagnostic formulation for anosognosia in Alzheimer's disease. J Neurol, Neurosurg Psychiatry. 2006;77(6):719-25.

15. Marshall GA, Kaufer DI, Lopez OL, Rao GR, Hamilton RL, Dekosky ST. Right prosubiculum amyloid plaque density correlates with anosognosia in Alzheimer's disease. J Neurol, Neurosurg Psychiatry 2004;75(10):1396-1400.

16. Dourado M, Laks J, Rocha M, Soares C, Leibing A, Engelhardt E. Consciência da doença na demência: resultados preliminares em pacientes com doença de Alzheimer leve e moderada. Neuropsiquiatria 2005;63(1):114-8.

17. Orfei MD, Varsi AE, Blundo C, Celia E, Casini AR, Caltagirone $\mathrm{C}$, et al. Anosognosia in mild cognitive impairment and mild Alzheimer's disease: frequency and neuropsychological. Am J Geriatr Psychiatry 2010;18(12):1133-40.

18. Kashiwa Y, Kitabayashi Y, Narumoto J, Nakamura K, Ueda H, Fukui K. Anosognosia in Alzheimer's disease: association with patient characteristics, psychiatric sympytoms and cognitive deficits. Psychiatry Clin Neurosci 2005;59(6):697-704

19. Starkstein SE, Sabe L, Chemerinski E, Jason L, Leiguarda R. Two domains of anosognosia in Alzheimer's disease. J Neurol, Neurosurg Psychiatry 1996;61(5):485-90.

20. Brucki SMD, Nitrini R, Caramelli P, Bertolucci PHF, Okamoto IH. Sugestões para o uso do miniexame do estado mental no Brasil. Neuropsiquiatria 2003;61(3B):777-81.

21. Morris JC. The Clinical Dementia Rating (CDR): current version and scoring rules. Neurology 1993;43(11):2412-4.

22. Riberto M, Miyazaki MH, Jucá SSH, Sakamoto H, Pinto PPN, Battistella LR. Validação da versão brasileira da medida de independência funcional. Acta Fisiátrica 2004;11(2):72-6 
23. Ricci NA, Kubota MT, Cordeiro RC. Concordância de observações sobre a capacidade funcional de idosos em assistência domiciliar. Rev Saúde Pública 2005;39(4):655-62.

24. Luzardo AR, Gorini MIPC, Silva APSS. Características de idosos com Doença de Alzheimer e seus cuidadores: uma série de casos em um serviço de neurogeriátria. Texto \& Contexto Enferm 2006;15(4):587-94.

25. Talmelli LFS, Gratão ACM, Kusumota L, Rodrigues RAP. Nível de independência funcional e déficit cognitivo em idosos com Doença de Alzheimer. Rev Esc Enferm USP 2010;44(4):933-9.

26. Lopes MA, Bottino CMC. Prevalência de demência em diversas regiões do mundo. Arq Neuropsiquiatr 2002;60(1):61-9.

27. Aprahamian I, Martinelli JE, Yassuda MS. Doença de Alzheimer: revisão de epidemiologia e diagnóstico. Rev Soc Bras Clín Méd 2009;7(1):27-35.

28. Cruz MN, Hamdan AC. O impacto da Doença de Alzheimer no cuidador. Psicol Estud 2008;13(2):223-9.
29. Celich KLS, Batistella M. Ser cuidador familiar do portador de Doença de Alzheimer: vivências e sentimentos desvelados. Cogitare Enferm 2007;12(2):143-9.

30. Reis RB, Cologna AJ, Martins ACP, Paschoalin EL, Junior ST, Suaid HJ. Incontinência urinária no idoso. Acta Cir Bras 2003;18(5):47-51.

31. Agnew SK, Morris RG. The heterogeneity of anosognosia for memory impairment in Alzheimer's disease: a review of the literature and a proposed model. Aging Ment Health 1998;2(1):7-19.

32. Borges SD, Radanovic M, Forlenza O. Fear of falling and falls in older adults with mild cognitive impairment and Alzheimer's disease. Aging Neuropsychol Cogn 2014;3:1-10.

33. Lenardt MH, Silva SC, Willig MH, Seima MD. O idoso portador da doença de Alzheimer: o cuidado e o conhecimento do cuidador familiar. REME Rev Min Enferm 2010;14(3):301-07. 